\title{
Vacuum inversion and securing of distal colonic pseudodiverticula with novel spiked O-rings
}

\author{
Giuseppe Amato, MD, ${ }^{1}$ Tudor Birsan, MD, ${ }^{2}$ Erna Granic, MD ${ }^{2}$ Gerd Silberhumer, MD, ${ }^{2}$ \\ Giovanni Cassata, MVD, ${ }^{3}$ Antonino Agrusa, MD, ${ }^{1}$ Roberto Puleio, MVD, ${ }^{3}$ Giorgio Romano, MD, ${ }^{1}$ \\ Susanna Lang, MD, ${ }^{4}$ Wayne Noda, BSBME, ${ }^{5}$ Christoph Gasche, MD $^{6}$ \\ Palermo, Italy; Vienna, Austria; Irvine, California, USA
}

Background: Diverticular disease is increasingly prevalent in Western societies and is associated with significant morbidity.

Objective: Two-stage endoscopic device development for inversion and secured ligation of colonic diverticula; first, human cadaver studies were performed to measure forces required for diverticular inversion; second, a novel set of devices (elastic spiked O-ring with delivery system) was tested in animals.

Design: Prospective, observational study of human cadavers and prospective, interventional study of a porcine model.

Setting: University hospital pathology laboratory and animal facility.

Intervention: Full-thickness inversion of the colonic wall with a pipelike delivery instrument to produce an inverted pseudodiverticulum that was secured with a spiked O-ring.

Main Outcome Measurements: The forces required for diverticular inversion, the secured closure of inverted pseudodiverticula, and the time until necrotic tissue falls off.

Results: A total of 248 of 248 of cadaveric sigmoid diverticula could be inverted by means of vacuum or forceps. The forces required for inversion ranged from 0.28 to $0.47 \mathrm{~N}$ (median, $0.37 \mathrm{~N}$ ). Twenty-four spiked O-rings were delivered in 6 living pigs to produce 24 inverted pseudodiverticula. One animal died the day after the procedure of a pulmonary thromboembolism. In the remaining 5 pigs, all delivered spiked O-rings remained in place for 7 to 22 days. At necropsy, none of the inverted sites showed signs of perforation but rather full-thickness reparative scarring with ingrowth of connective tissue.

Limitations: Animal model, stiff pipelike delivery instrument, variations in diverticular location, diameter, and size.

Conclusions: Endoluminal inversion and securing of colonic diverticula induces tissue necrosis, diverticular sloughing, and full-thickness scarring.

Diverticular disease has become an increasingly prevalent health problem in Western societies that affects men and women to a similar extent. This has been attributed

DISCLOSURE: Drs Amato and Gasche are consultants for and Dr Noda is an employee of Insightra. All other authors disclosed no financial relationships relevant to this article.

Copyright $\odot 2015$ by the American Society for Gastrointestinal Endoscopy 0016-5107/\$36.00

http://dx.doi.org/10.1016/j.gie.2014.09.051

Received January 10, 2014. Accepted September 19, 2014.

Current affiliations: Department of General Surgery and Urgency, University of Palermo, Palermo, Italy (1), Department of General Surgery, to population aging, colonic intraluminal pressure, and insufficient fiber intake. ${ }^{1,2}$ Adverse events such as diverticulitis and bleeding develop in a significant number of 
patients. Open or laparoscopic surgery remains the standard of care for recurrent cases. ${ }^{3}$ It is not uncommon, however, that advanced patient age or concomitant disease represents a limitation to such an approach.

Early colonoscopy is feasible for acute diverticulitis, and various endoscopic interventions have been evaluated for diverticular hemorrhage. ${ }^{4-6}$ In addition to epinephrine injection, thermal coagulation, and hemoclipping, endoscopic band ligation has been reported to be safe and effective for the treatment of diverticular hemorrhage. $^{7,8}$ The rebleeding rate after epinephrine injection or thermal coagulation is considerable, ${ }^{6}$ and endoscopic placement of a hemoclip at a bleeding vessel may be difficult in cases of a small diverticular orifice or bleeding from the diverticular dome. Thus, endoscopic band ligation has been proposed as the treatment of choice by some authors. ${ }^{7-10}$ Several issues, however, limit the use of endoscopic band ligation for diverticular bleeding. First, current band ligation devices were developed for the treatment of esophageal varices and are not approved for use in colonic diverticular bleeding. Second, immediate or subsequent slippage of the O-ring in the course of colonic peristalsis has been reported. ${ }^{10}$ Third, the size and diameter of suction cup are not adjusted to the diverticular orifice. In addition, complete diverticular inversion with effective contact of all tissue segments is the premise for sustained healing.

Here we report on (1) human cadaver studies to evaluate the diameter and size as well as the forces required to invert diverticula and on (2) the development of a novel set of devices for vacuum inversion and securing of distal colonic pseudodiverticula in a porcine model.

\section{MATERIALS AND METHODS}

\section{Human cadaver studies}

The study was approved by the Ethics Committee of the Medical University of Vienna. Sigmoid colon specimens were excised and studied on the bench immediately after the postmortem examination at the Department of Pathology, General Hospital of Vienna. The identity of the corpse was blinded to the investigators. The distribution of diverticula over the sigmoid colon and the morphologic characteristics of the diverticula were determined. Thereafter, diverticula were inverted, and the forces required for inversion were measured. Measurements included length of sigmoid colon (in centimeters), the number of diverticula, the distance between single diverticula (in millimeters), and the diameter, depth, orifice, and wall thickness of each diverticulum (in millimeters). All measurements were accomplished by using a digital caliper (CEN-TECH Pittsburgh; Camarillo, Calif; accuracy $\pm 0.02 \mathrm{~mm}$ ) with readings from 0 to $150 \mathrm{~mm}$. The forces required for inversion of diverticula were measured by a hanging scale (Digipen 500; American Weigh Scales, Inc, Norcross, Ga). After ef-

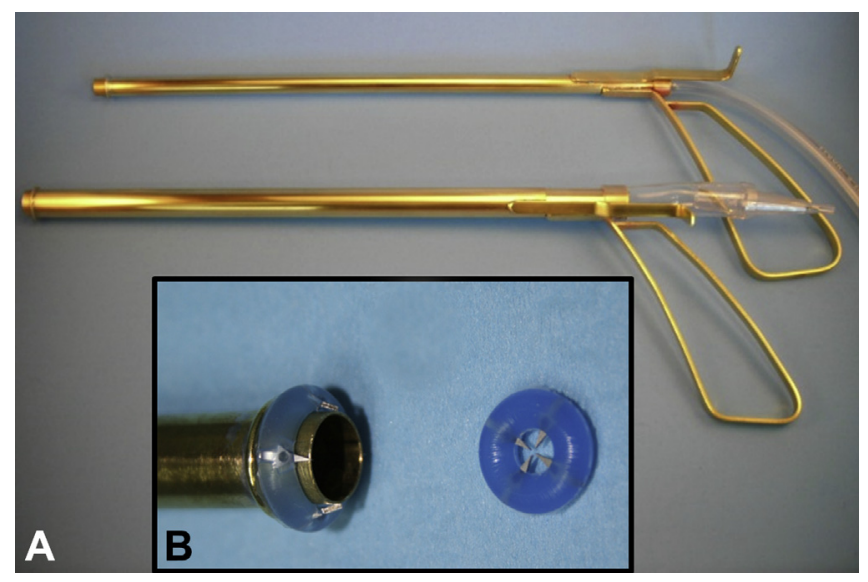

Figure 1. A, The tool set for the animal experiments. Note the pipelike delivery device. $\mathbf{B}$, The shape of the spiked O-rings with 1 ring loaded on the edge of the delivery device before the procedure.

fective inversion of diverticula to the luminal side, the characteristics of the inverted pouch were determined (diameter, height, and thickness in millimeters).

\section{Devices}

A proprietary pipelike delivery instrument was connected with a standard vacuum pump to invert a segment of the colonic wall. This instrument comprises 2 closely fitted concentric tubes (Figs. 1A and B), which had an outer diameter of $12.2 \mathrm{~mm}$, and allows deployment of the tissue-securing ring. Such a ring was made from silicone rubber to capture the inverted pseudodiverticulum. To secure the grip on the tissue at the base the inverted pseudodiverticulum, the ring also includes 4 metal spikes (Fig. 1B). The elastomeric properties of the ring serve to direct and drive the integral spikes into the tissue. The spikes in the rings were manufactured from stainless steel, which allows radiographic detection. The rings were pressure-tested on pig bowel in excess of $200 \mathrm{~mm}$ $\mathrm{Hg}$.

\section{Porcine model of full-thickness colonic wall inversion}

Animal experiments were conducted at the animal facility of the Experimental Zooprophylactic Institute A. Mirri, Palermo, Italy, in compliance with the guidelines of the Animal Care Protocol for Experimental Surgery issued by the Italian Ministry of Health. The study was carried out on six 3- to 4-month-old female pigs weighing between 35 and $45 \mathrm{~kg}$ while they were under general anesthesia.

\section{Surgical procedure}

Four colonic areas were inverted in each pig, resulting in 4 inverted pseudodiverticula: 2 in the intraperitoneal colon and 2 in the extraperitoneal rectocolic segment (Fig. 2A). To assess the distal intraperitoneal colon 

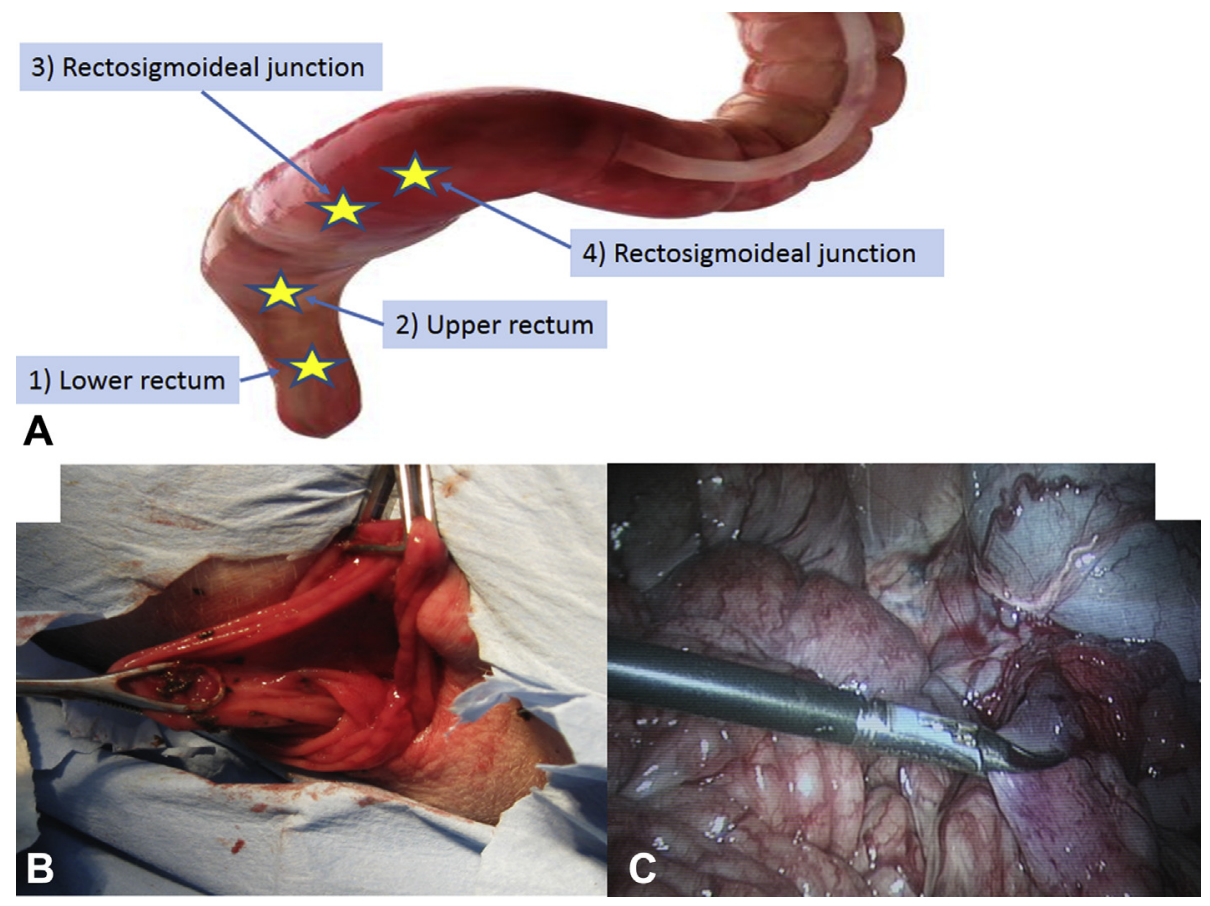

Figure 2. A, Position of the O-rings in the porcine model (yellow stars). B, The transanal pull-through maneuver to exteriorize the intra-abdominal intestine. $\mathbf{C}$, The combined video-assisted exteriorization of the intra-abdominal colon.

with the pipelike delivery instrument from the anus, a transanal pull-through of the lower intestine was performed. This was achieved by laparoscopically assisted intussusception of the lowest segments of the intraabdominal colon (Figs. 2B and C).

The colonic wall area was inverted by suction with the pipelike delivery instrument and a standard vacuum pump (Fig. 3A). This maneuver resulted in a pouch that mimicked an inverted pseudodiverticulum that was secured by the spiked O-ring (Fig. 3B). The capturing of this pouched intestinal wall was intended to be full thickness including all layers of the intestinal wall. The tissue-securing ring with the 4 spikes was specifically shaped to apply adequate strangling forces to achieve an irreversible ischemia within a few days, resulting in necrosis of the incarcerated pouched tissue. Once the necrosis was achieved, the ischemically altered pouch fell off into the lumen of the colon. In 5 of the 6 animals, the inverted pseudodiverticulum was pierced to introduce the straight barbell just above the ring (Fig. 3C). In 17 of 24 pseudodiverticula, the full apex of the inverted pseudodiverticula was completely excised (Fig. 3D) and sent to a pathologist for histological study. The sites were marked with Prolene stitches; starting proximally, the stitches were secured with 1 to 4 knots to better identify the sites of the previous O-ring deployment at necropsy. The secured closure and the time needed for the necrosis and the pouch falling off were the primary endpoints of the study (Fig. 4A). Finally, the colon was returned into the peritoneal cavity under laparoscopic visualization, thereby establishing a normal visceral outline (Fig. 4B), and the sites of the intraperitoneal inverted pseudodiverticula were captured photographically (Fig. 4C). The appropriate placement of the rings was also documented by plain radiographs of the lower abdomen in 2 projections carried out immediately after closing the procedure (Figs. 4D and $\mathrm{E})$.

All animals received a postoperative antibiotic treatment with doxycycline $(8 \mathrm{mg} / \mathrm{kg} /$ day) for 3 days. Postoperative radiographs were obtained every week until no more spiked O-rings were visible. At this stage, the animals were euthanized, and the distal colon and the rectum were removed (Fig. 5B) and opened to detect the Prolene knots marking the sites of the inverted pseudodiverticula (Fig. 5C), which were excised for histological evaluation. Tissues were fixed with $10 \%$ phosphate-buffered formalin and embedded in paraffin. Sections were cut at 4 to $6 \mu \mathrm{m}$ and stained with hematoxylin-eosin, Azan-Mallory, and Weigert van Gieson stains.

\section{Statistical analysis}

SPSS Version 16.0 (SPSS Inc, Chicago, Ill) was used for all statistical calculations. Descriptive methods were used to describe characteristics of noninverted and inverted diverticula. If not stated otherwise, continuous variables are expressed as mean (range). Correlations between continuous variables were determined by the Pearson correlation. All statistical tests were 2 tailed, and statistical significance was accepted if the $P$ value was $<.05$. 


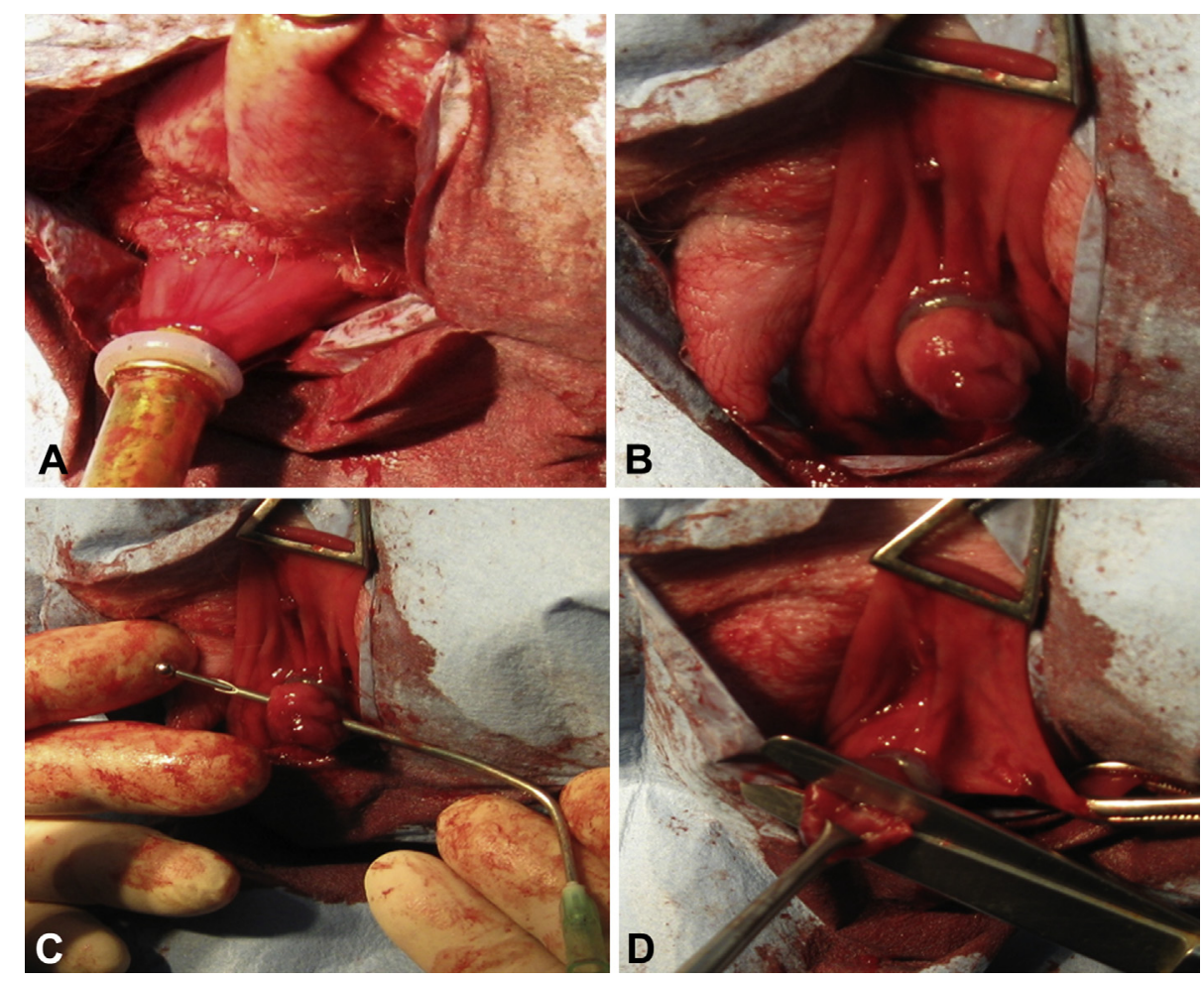

Figure 3. A, Suction applied by the colonic wall before delivery of the spiked O-ring. B, The spiked O-ring in place with a newly formed inverted pseudodiverticulum. C, Piercing the apex of the inverted pseudodiverticulum with a straight barbell. D, Full-thickness excision of the apex of the pseudodiverticulum.

\section{RESULTS}

\section{Human cadaver studies}

Thirty sigmoid colons from Caucasian corpses carrying at least 1 diverticulum were examined on the bench. In total, 248 diverticula were investigated, between 3 and 13 per specimen (mean, 8 ). The mean length of the sigmoid specimen was $52 \mathrm{~cm}$ (range $38-69 \mathrm{~cm}$ ). The shortest distance between 2 single diverticula was $7 \mathrm{~mm}$ (mean, $2-5 \mathrm{~mm}$ ), and the longest distance was $42 \mathrm{~mm}$ (mean, 9-68 mm). The mean diverticular diameter was $8.0 \mathrm{~mm}$ (range 2.8-14.8 mm), the depth was $9.2 \mathrm{~mm}$ (range 2.9-14.5 $\mathrm{mm}$ ), the orifice diameter was $5.9 \mathrm{~mm}$ (range $0.1-13.8 \mathrm{~mm}$ ), and the wall thickness was $3.1 \mathrm{~mm}$ (range 0.2-3.9 $\mathrm{mm}$ ).

A total of 248 of 248 diverticula could be inverted by means of a vacuum or forceps. The force required to invert diverticula ranged from 0.28 to $0.47 \mathrm{~N}$ (median, $0.37 \mathrm{~N}$ ). Both the diverticular diameter and its depth related to the required inversion forces $\left(R^{2}=0.836\right.$, $P<.001$ and $R^{2}=0.662, P<.001$, by the Pearson correlation, respectively). After inversion, 145 of 247 diverticula (59\%) remained inverted, whereas the other 102 (41\%) popped back into the noninverted position. The diameter of the inverted diverticular pouch was between 2.6 and $14.3 \mathrm{~mm}$ (mean, $7.2 \mathrm{~mm}$ ), the height was between 2.8 and $13.4 \mathrm{~mm}$ (mean, $8.3 \mathrm{~mm}$ ), and the thickness at the base was between 1.8 and $3.9 \mathrm{~mm}$ (mean, $2.9 \mathrm{~mm}$ ). Based on these measurements, the inversion of diverticula by means of the pipelike delivery instrument with a standard endoscopic vacuum pump system was considered feasible.

\section{Porcine model for full-thickness colonic wall inversion}

A total of 24 spiked O-rings were delivered in 6 animals to produce 24 pouches, so-called inverted pseudodiverticula. Twelve were delivered in the intraperitoneal part of the distal colon and 12 in the rectum. The O-ring placement in the upper colon was assisted by laparoscopy. After the placement of the spiked O-rings and the full-thickness excision of the apex of the inverted pseudodiverticula, no intestinal perforation occurred. Histological examination of the excised apex supported the notion of full-thickness excision. All pigs survived the surgical procedure except 1 animal, which died the day after the intervention of a pulmonary thromboembolism. Nevertheless, despite this seemingly unsuccessful outcome, this event allowed us to detect the effectiveness of the strangling force of the spiked O-rings during autopsy. In fact, all 4 inverted pseudodiverticula were clearly necrotic 24 hours after the 


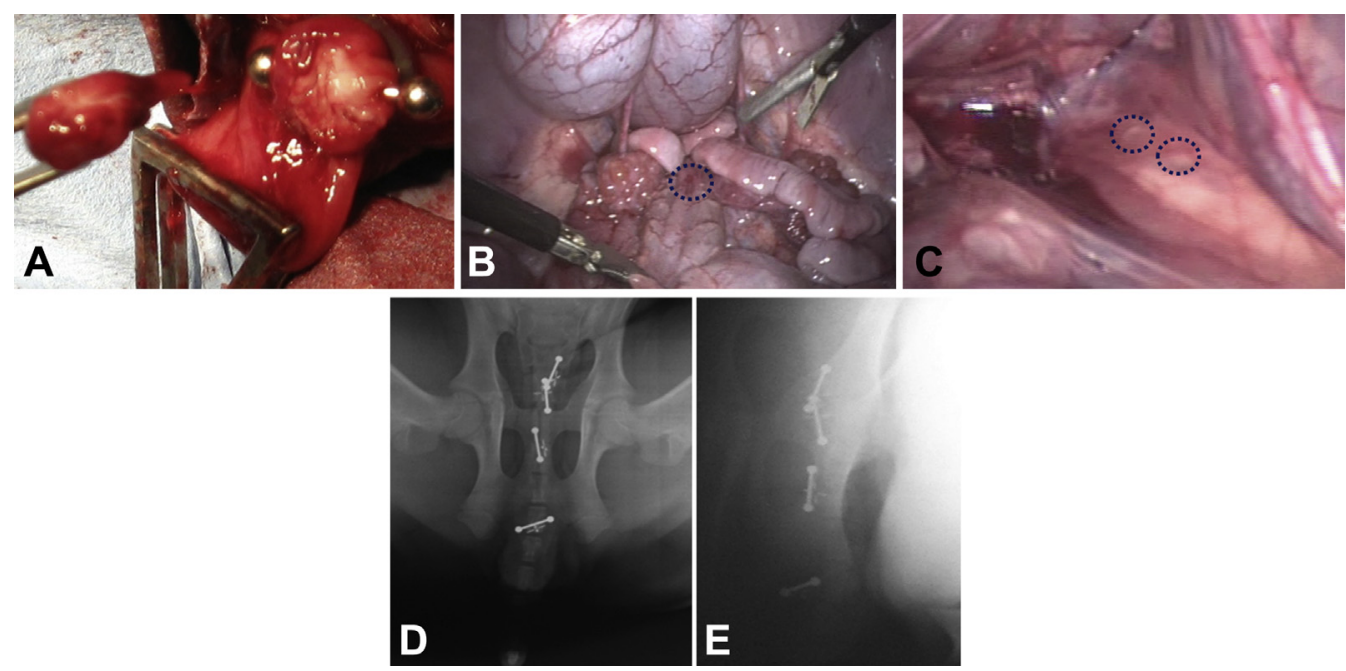

Figure 4. A, The pseudodiverticulum at the end of the procedure. The spiked O-ring is in place, just behind the barbell. Note the full-thickness perforation of the tip of the pseudodiverticulum. B, The intra-abdominal colon being repositioned by means of laparoscopic forceps after placement of the spiked O-rings. The blue ring marks the place where the colonic wall was inverted to form the pseudodiverticulum. C, Intra-abdominal view of hollows demonstrating the inversions of the intestinal wall (blue rings). D, Postoperative radiographic detection of the O-rings, anteroposterior image. E, Postoperative radiographic detection of the O-rings, lateral image.

procedure (Fig. 5A). This was evident macroscopically as well as microscopically.

In the remaining 5 pigs, all of the delivered 20 spiked O-rings remained in place at least 1 week, with 12 rings ejected between days 7 and 14, 7 between days 14 and 21. One spiked O-ring was ejected beyond the third week after implantation (Table 1). The autopsy was performed after radiographs demonstrated that all O-rings had fallen off. At macroscopic examination of the distal colon and rectum, all suture marks were found in place except in 2 pigs where 1 suture mark (the most proximal with only 1 Prolene knot) was missing (Fig. 5C). The sites where the inverted pseudodiverticula were located showed a small dip covered by scar tissue (Fig. 5C). All 20 pseudodiverticula had been ejected, and the sites that were covered by newly ingrown scar tissue were all excised for histological study. On histological examination, all tissue samples from the sloughed sites displayed an inflammatory infiltrate with lymphocytes and neutrophils (Fig. 6A). The colonic mucosal surface showed the ingrowth of connective tissue covering the basal elements of the mucosa (Fig. 6B). These components were interspersed among connective fibers and newly formed vascular structures (Fig. 6C). The junction between the tissue layers of the colonic wall was visibly evident, shaping complete full-thickness reparative scarring (Fig. 6D).

\section{DISCUSSION}

Despite advances in imaging technology and medical treatment, the increasing prevalence of diverticular disease in developed countries is associated with significant morbidity and mortality. Although more recent studies have downscaled the estimates of lifetime risk of the development of diverticulitis from $25 \%{ }^{11,12}$ to $4 \%,{ }^{13}$ it remains a major burden of disease for our society. Mesalamine and rifaximin have been proposed for prevention of diverticulatis ${ }^{14-17}$; however, larger controlled trials on mesalamine had negative results, ${ }^{18}$ leaving laparoscopic or open surgery as the remaining option.

A potential minimally invasive approach for the treatment of colonic diverticula is endoscopic band ligation. Band ligation has been successfully introduced in patients with diverticular bleeding. ' For the use of diverticular removal, however, complete diverticular inversion with serosa-to-serosa contact, temporal securing of ligation bands, and full-thickness scarring before such bands fall off are required. Current bands were not developed for such use, and immediate or subsequent slippage of the O-ring in the course of peristalsis is a likely event. In our cadaver study, measurements and inversion tests revealed that $50 \%$ of cases of diverticular disease of the sigmoid colon have only 3 to 8 diverticula, a number that seems accessible for an endoluminal approach, but may be higher in patients with recurrent diverticultis. The orifice diameter and depth of diverticula showed high variation, indicating the need for different sizes of suction cups and O-rings. On the bench, all diverticula could be inverted, and the forces needed were $<0.5 \mathrm{~N}$, which can be achieved with a standard vacuum pump.

The specific design of the spiked O-ring should enable serosa-to-serosa contact and prevent slippage of the O-ring before tissue scarring was complete. To prove that actual sloughing of the inverted pseudodiverticula, we 


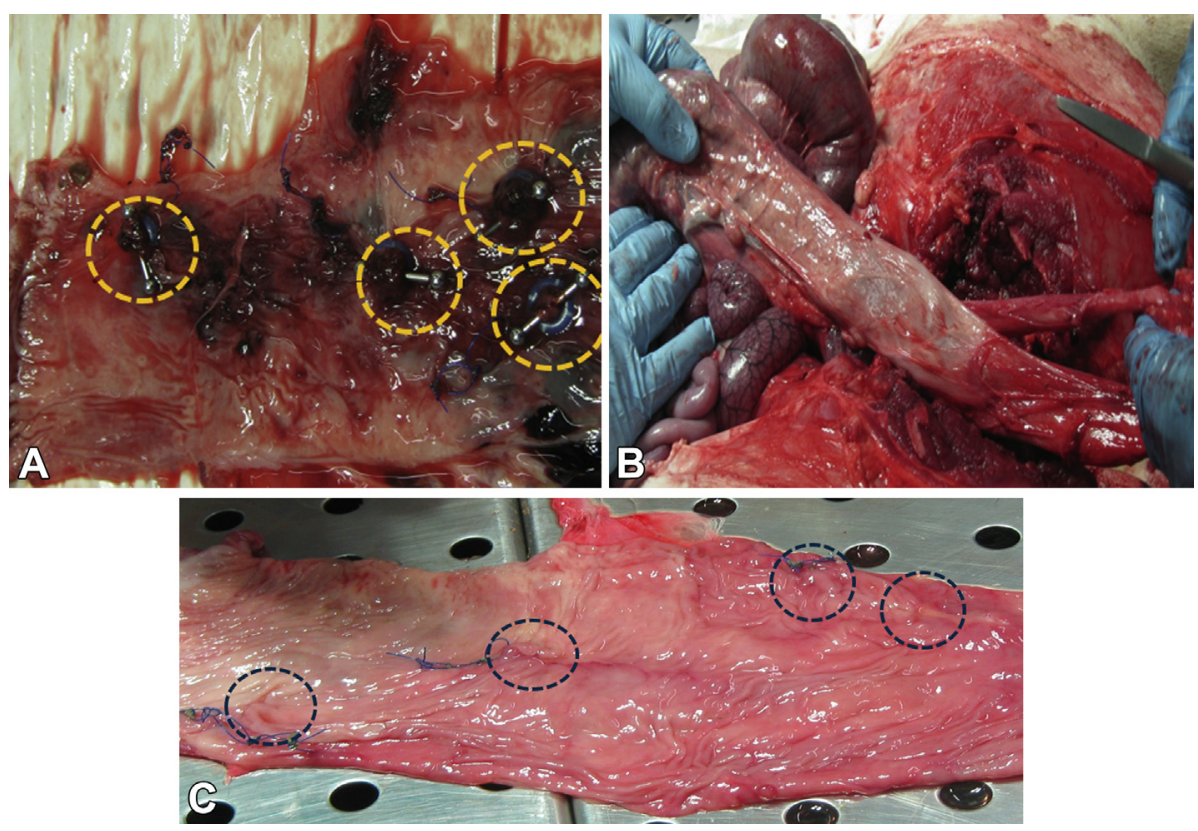

Figure 5. A, Coloanal segment excised 24 hours after placement of 4 spiked O-rings. All pseudodiverticula show clear signs of necrosis but with spiked O-rings, barbells, and suture marks in place. B, Resected coloanal segment during autopsy 16 days after the surgical procedure. C, Internal view of the coloanal segment at autopsy. Four dips at the site of the ejected pseudodiverticula are clearly visible and covered by scar tissue (blue rings). The suture mark at the most proximal pseudodiverticulum (to the right) is missing.

\begin{tabular}{|lccccccc|}
\hline $\begin{array}{l}\text { TABLE 1. Results summary of the experimental testing } \\
\text { in the porcine model }\end{array}$ & \multicolumn{7}{c|}{ Pigs } \\
\cline { 2 - 8 } & $\mathbf{1}$ & $\mathbf{2}$ & $\mathbf{3}$ & $\mathbf{4}$ & $\mathbf{5}$ & $\mathbf{6}$ \\
& 12 & 15 & 14 & 16 & 13 & 14 \\
\hline Age, wk & 35 & 42 & 44 & 45 & 38 & 43 \\
Weight, kg & 4 & 4 & 4 & 4 & 4 & 4 \\
\hline Spiked O-rings, no. & 0 & 4 & 4 & 4 & 4 & 4 \\
\hline Barbells, no. & 0 & 4 & 2 & 4 & 3 & 4 \\
\hline Apex excisions, no. & 0 & 7 & 7 & - & 8 & 8 \\
\hline First O-ring off* & 8 & 15 & 22 & 12 & - & 14 & 16 \\
\hline Last O-ring off* & 16 & 22 & 15 & 1 & 15 & 16 \\
\hline Autopsy* & - & - & - & Death & - & - \\
\hline Adverse events & & & & & \\
\hline *Days after procedure. & & & & &
\end{tabular}

introduced a barbell behind the spiked O-ring and perforated the apex of the inverted pseudodiverticula. This way it was impossible for the spiked O-ring to slip before the inverted pseudodiverticula had fallen off. Five of 6 pigs survived the procedure, and none of the inverted sites showed any sign of perforation. Full-thickness reparative scarring was identified by histology at all inversion sites. One pig had died early in the study of a thromboembolic event that was the result of the general surgical procedure rather than the inversion of the pseudodiverticula. We conclude that inversion of colonic tissue can be secured with such spiked O-rings, which remain in place for as long as 22 days until sufficient healing. The endoluminal removal of diverticula may become feasible in future.

There are several limitations to our study. The inverted pseudodiverticula in pigs comprise all layers of the bowel wall (including the muscular layer). Colonic diverticula in humans, however, are false diverticula that do not involve the muscularis propria. By removing the sigmoid colon from the abdomen to measure the inversion forces in human cadavers, we have likely underestimated the actual forces because the abdomen is a closed space and vacuum forces are at play. The same is likely true for creation of the pseudodiverticula in pigs because laparoscopy assisted this procedure. Also the intracolonic pressure in young pigs likely differs from that in elderly humans. Tests were performed only in the very distal part of the colon, which has thicker wall layers. More proximal colonic parts are thinner with different motility dynamics. Concerning the porcine model used in our study, we are well aware that there is not an ideal animal model that could adequately mimic the structure of colonic diverticula in humans. Nevertheless, we are confident that the model is comparable to humans regarding structural similarities as well as the biological response. Further limitations are related to the stiff delivery system, which needs adaption for flexible endoscopes, and to the different diverticular sizes. Last, luminal stenosis often occurs with presence of multiple diverticula, which may cause luminal obstruction when inverted. Also, one may expect disease recurrence in temporal and regional proximity. The complexity of issues is 

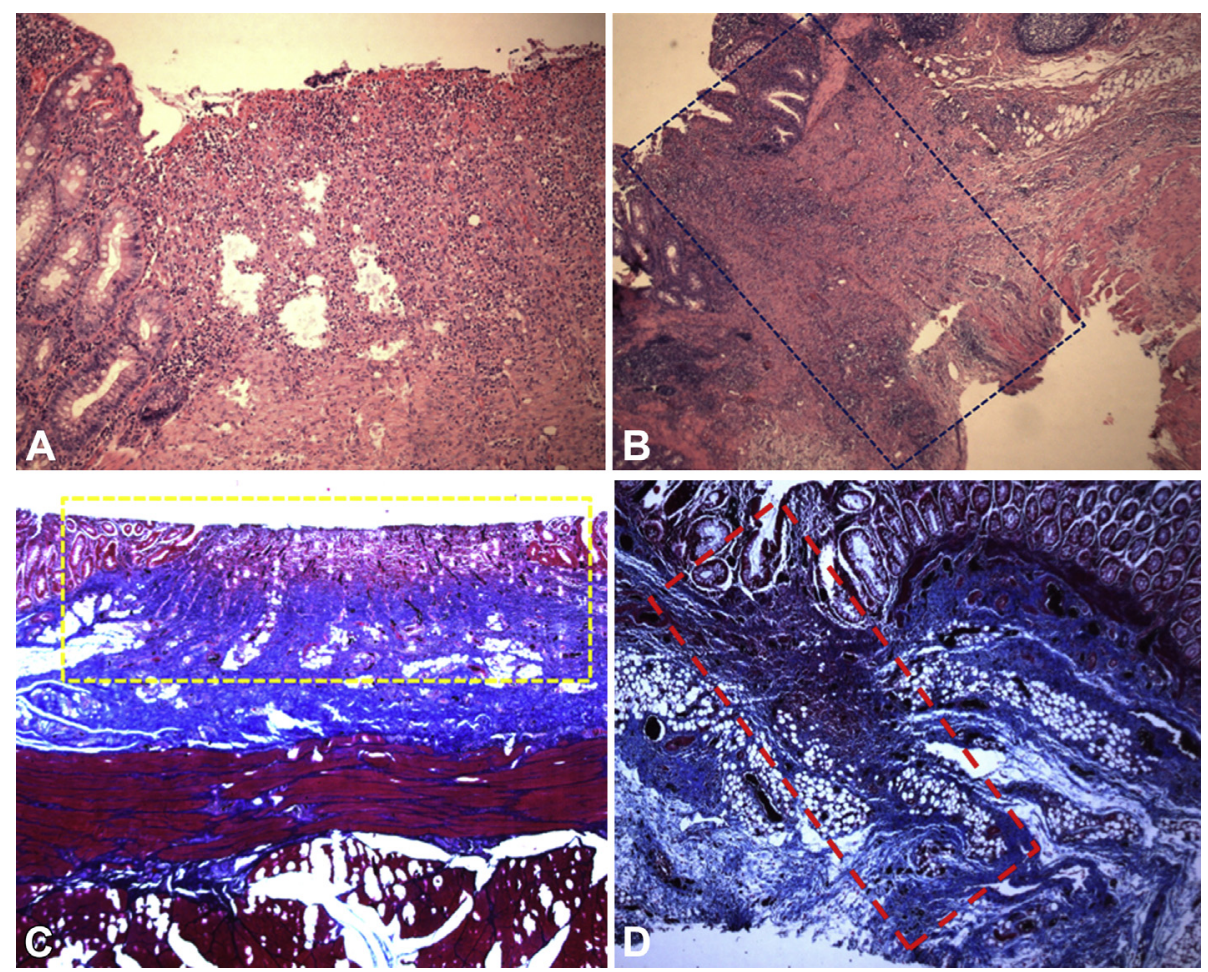

Figure 6. A, Transition zone at the edge of the sloughed pseudodiverticulum 15 days after the procedure: in-growth of inflammatory infiltrate and connective tissue close to normal intestinal mucosa (H\&E, orig. mag. $\times 10)$. B. Tissue specimen excised from the base of the sloughed pseudodiverticulum 16 days after the procedure. Full-thickness transition zone (blue rectangle) between the 2 layers of the intestine where the sloughed pseudodiverticulum was placed. Evident reparative process is in progress with newly formed scar tissue and inflammatory infiltration (H\&E, orig. mag. $\times 5$ ). C, Transition zone (yellow rectangle) at the base of the sloughed pseudodiverticulum 16 days after the procedure: ingrowth of connective tissue and neoangiogenesis between the 2 margins of the healthy mucosa (Azan Mallory, orig. mag. $\times 5$ ). D, Full-thickness transition zone (red rectangle) indicating connective ingrowth and neoangiogenesis to form reparative scar tissue after sloughing of the pseudodiverticulum. The 2 segments of the intestinal wall are fully integrated (Azan Mallory, orig. mag. ×5).

definitely limiting to an immediate clinical development of such procedure in the near future.

\section{REFERENCES}

1. Strate LL. Diverticulosis and dietary fiber: rethinking the relationship. Gastroenterology 2012;142:205-7.

2. Burkitt DP, Walker AR, Painter NS. Effect of dietary fibre on stools and the transit-times, and its role in the causation of disease. Lancet 1972;2:1408-12.

3. Wong WD, Wexner SD, Lowry A, et al. Practice parameters for the treatment of sigmoid diverticulitis-supporting documentation. The Standards Task Force. The American Society of Colon and Rectal Surgeons. Dis Colon Rectum 2000;43:290-7.

4. Lahat $A$, Yanai $H$, Menachem $Y$, et al. The feasibility and risk of early colonoscopy in acute diverticulitis: a prospective controlled study. Endoscopy 2007;39:521-4.

5. Jensen DM, Machicado GA, Jutabha R, et al. Urgent colonoscopy for the diagnosis and treatment of severe diverticular hemorrhage. N Engl J Med 2000;342:78-82.

6. Bloomfeld RS, Rockey DC, Shetzline MA. Endoscopic therapy of acute diverticular hemorrhage. Am J Gastroenterol 2001;96:2367-72.

7. Farrell JJ, Graeme-Cook F, Kelsey PB. Treatment of bleeding colonic diverticula by endoscopic band ligation: an in-vivo and ex-vivo pilot study. Endoscopy 2003;35:823-9.

8. Tucker LE. Diverticular bleeding: novel treatment with band ligation. Mo Med 2004;101:61-3.
9. Setoyama T, Ishii N, Fujita Y. Endoscopic band ligation (EBL) is superior to endoscopic clipping for the treatment of colonic diverticular hemorrhage. Surg Endosc 2011;25:3574-8.

10. Ishii N, Setoyama T, Deshpande GA, et al. Endoscopic band ligation for colonic diverticular hemorrhage. Gastrointest Endosc 2012;75:382-7.

11. Parks TG. Natural history of diverticular disease of the colon. Clin Gastroenterol 1975;4:53-69.

12. Stollman N, Raskin JB. Diverticular disease of the colon. Lancet 2004;363:631-9.

13. Shahedi K, Fuller G, Bolus R, et al. Long-term risk of acute diverticulitis among patients with incidental diverticulosis found during colonoscopy. Clin Gastroenterol Hepatol 2013;11:1609-13.

14. Tursi A, Brandimarte G, Daffina R. Long-term treatment with mesalazine and rifaximin versus rifaximin alone for patients with recurrent attacks of acute diverticulitis of colon. Dig Liver Dis 2002;34: 510-5.

15. Brandimarte G, Tursi A. Rifaximin plus mesalazine followed by mesalazine alone is highly effective in obtaining remission of symptomatic uncomplicated diverticular disease. Med Sci Monit 2004;10:PI70-3.

16. Di Mario F, Aragona G, Leandro G, et al. Efficacy of mesalazine in the treatment of symptomatic diverticular disease. Dig Dis Sci 2005;50:581-6.

17. Stollman N, Magowan S, Shanahan F, et al. A randomized controlled study of mesalamine after acute diverticulitis: results of the DIVA trial. J Clin Gastroenterol 2013:47:621-9.

18. Kruis W, Meier E, Schumacher M, et al. Randomised clinical trial: mesalazine (Salofalk granules) for uncomplicated diverticular disease of the colon-a placebo-controlled study. Aliment Pharmacol Ther 2013;37: 680-90. 